\title{
Visual cells in excised Limulus eyes: 2. Excision does not reduce sensitivity to light
}

\author{
GERALD S. WASSERMAN \\ Purdue University, West Lafayette, Indiana
}

\begin{abstract}
Excision of the lateral eye of Limulus is believed to compromise it by greatly reducing its sensitivity to light stimuli. This belief derives from an experiment in which excised eyes were studied in the daytime and intact eyes were studied at night. But since a circadian elevation of nighttime sensitivity occurs in this eye, the belief is based on a confounded experiment in which like was not compared with like. In the present paper, like is compared with like; light sensitivity is assessed by comparing the intensity-response curves of receptor potentials that were recorded intracellularly from excised and from intact eyes. These data clearly indicate that the excised eye is not less sensitive than the intact eye. They further indicate that this situation exists for periods of time that exceed $5 \mathrm{~h}$ after excision, which means that this preparation provides ample time to replicate behaviorally interesting experiments while recording from a single neuron.
\end{abstract}

In order to avoid certain persistent errors that arise in comparing brain and behavior (see Wasserman, 1991; Wasserman \& Kong, 1974), it is useful to have physiological preparations that last long enough to replicate the conditions used in behavioral experiments. The excised lateral eye of the horseshoe crab, Limulus, is ideal in such studies, for three reasons. First, intracellular microelectrodes can be easily inserted into photoreceptor and optic nerve cells in an eye that can be excised and maintained in ordinary artificial sea water for up to $24 \mathrm{~h}$. Second, in our hands, this preparation provides cellular recordings that remain in stable condition for enough time to do detailed experiments with care and precision. We routinely record intracellularly from cells that maintain constant stability for periods of up to $10 \mathrm{~h}$. Third, this preparation makes it possible to obtain simultaneous records from a receptor cell and from the particular optic nerve fiber that the particular receptor drives (Wang \& Wasserman, 1985).

Others believe, however, that the excised eye of Limulus is abnormal and contains only "remnants" of the "natural depolarizing potential" (Barlow \& Kaplan, 1977, pp. 218-219). We therefore previously published detailed information that demonstrated that normal physiological functions do exist in the excised eye (Wang \& Wasserman, 1980; Wang-Bennett \& Wasserman, 1991). Three separate physiological phenomena were easily demonstrated, despite the belief that they are vulnerable to excision; those data qualitatively indicated that function is quite normal in the excised eye.

However, it is also widely believed that quantitative changes occur upon excision-specifically, that they puta-

Correspondence should be sent to Gerald S. Wasserman, Sensory Coding Laboratory, Department of Psychological Sciences, Purdue University, West Lafayette, IN 47907-1364 (e-mail: codelab@psych.purdue.edu). tively reduce sensitivity to light. This view stems from an experiment done by Barlow and Kaplan (1971), who recorded extracellular spikes in afferent axons coming from an otherwise intact Limulus lateral eye. They compared these results with those obtained by using eyes that had been partially or completely isolated from the normal support of the animal.

The comparison assessed the sensitivities of these preparations to light flashes; it indicated that a sensitivity decline set in immediately after excision and that the decline continued progressively for hours. Several possible quantitations of the decline are possible; one quantitation yields a truly enormous decline, namely by a factor of about $10^{5}$. These data have led to the widely shared view that the sensitivity of excised eyes is hopelessly compromised.

This view is flawed, however, because it is not based on a comparison of like with like: the relative sensitivities of any two preparations can only be demonstrated by comparing recordings taken under conditions that are as nearly identical as possible. Unfortunately, this was not done by Barlow and Kaplan (1971). They did compare excised and intact eyes, but they did not compare like with like. Instead, they compared data recorded from excised eyes during the daytime with data recorded from intact eyes during the middle of the night. This difference will be obvious to anyone who inspects their Figure 1 with the time of day in mind; that variable can be quantitated by measuring the time between a measurement and midnightmeasures taken in the middle of the day would be $12 \mathrm{~h}$ away from midnight, at most, whereas measures taken in the middle of the night would approach a 0-h difference. Such an analysis indicated that the excised eyes were recorded in the daytime (mean absolute time between midnight and recording $=8.4 \pm 0.8 \mathrm{~h}$ ), but that the intact eyes were recorded at night (mean time between midnight and recording $=3.0 \pm 0.6 \mathrm{~h}$ ). This difference convincingly $[t(18)=93.1, p<.0001]$ demonstrates that a compari- 


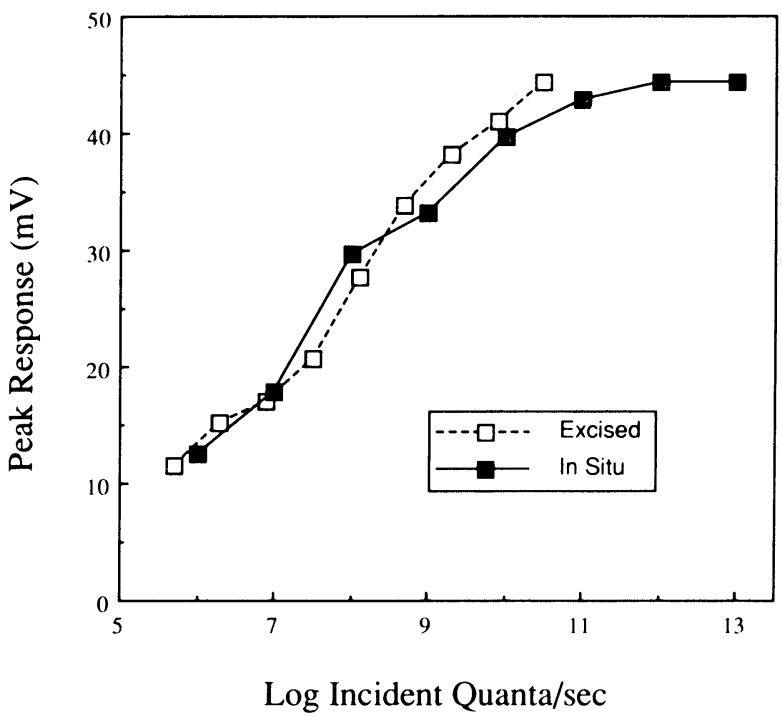

Figure 1. Size of intracellularly recorded photoreceptor cell responses to light flashes as a function of the intensity of the stimulus. Light intensity is given in terms of the number of quanta per second that are incident on the rhabdom, which contains the lightsensitive photopigment. Responses are measured by the depolarization of the cell that exists at the peak of the receptor potential evoked by a flash. Data for excised eyes come from a typical cell from the study reported in Wang-Bennett and Wasserman (1991), while the data for eyes in situ come from the best cell encountered by Barlow and Kaplan (1977).

son of like with like was not made by Barlow and Kaplan. (It must, of course, be understood that their experiment can now be seen to be confounded because later work showed that Limulus lateral eyes are more sensitive during the night than they are in the day; see Barlow, Chamberlain, \& Kaas, 1984.)

In Figure 1, a comparison of like with like is presented by means of intensity-response functions that derive from intracellular recordings from photoreceptor cells in the lateral eye. Two conditions are represented: one in which the eye is completely excised and immersed in a bath of seawater, and another in which the eye is relatively intact. The latter condition (labeled in situ) represents data recorded from unexcised eyes via an electrode inserted through a small hole in the cornea.

The plotted functions give the magnitude of the depolarization produced by the initial transient peak of the receptor potential, as a function of the rate of arrival of light quanta incident on the rhabdom (where the visual photopigment is located). The in situ data are taken directly from Figure 8 of Barlow and Kaplan (1977); this represents, according to them, one of their very best results. Although this particular cell was held for at least $8 \mathrm{~h}$, they say that the typical in situ cell could only be held for about $3 \mathrm{~h}$. Longer durations in situ recordings were subsequently obtained (Barlow, Kaplan, Renninger, and Saito, 1987); no evidence of a sensitivity increase was evident in this later study. (It will be appreciated that a variety of mechanical and vibratory problems exist in an intact animal that can quickly compromise an intracellular microelectrode's competence.) The time of day of this recording was not given.

The data for excised eyes come from Figure 3 of WangBennett and Wasserman (1991); they are from a cell that was held for more than 5 daytime hours in a strictly stable condition, as indexed by the stability of responses to constant check stimuli delivered throughout the experiment. It is important to understand that the data from this cell are not unrepresentative-they are quite typical of data taken from cells that satisfy this strict stability criterion; any such cell will usually be equally sensitive.

For technical reasons, the light stimulus was delivered in different ways in the two experiments. The in situ eye was stimulated with light coming through the cornea, whereas the excised eye was stimulated with light coming through a dark pigment that shields the receptor cells. Therefore the data from both experiments were expressed in terms of light quanta per ommatidium incident on the photopigment-containing rhabdom.

The plot provides a fair comparison of like with like. The data come from recordings from good cells, both of which lasted for hours. Comparison of like with like also requires the use of similar techniques; in both cases, glass micropipettes with similar resistances were used, and they were filled with the same electrolyte. Finally, comparison of like with like means using the same procedures. These were essentially the same, except as noted below.

It must be noted, however, that the comparison given in Figure 1 involves comparing data collected in one laboratory with data from another. Hence, it may be argued that the data have merely been made to appear equated, perhaps through inappropriate adjustments. But the opposite is the case. There are a number of ways in which the excised data could have been made to look more sensitive than the intact data. The plot given here is the result that is most favorable to the intact, rather than the excised, preparation, for the following reasons.

1. The choice of response measure could have been different because light evokes a response with a complex waveform: it has an initial transient peak, which sags down to a plateau or steady-state response. The plateau data are available from both sources, so they could have been used instead of the peak data given in Figure 1. But that comparison would have suggested that the excised eye is 1-2 log units more sensitive than the intact eye, not less. However, this measure was not used to construct Figure 1, because the plateau intensity-response functions are much shallower than those based on the peak and hence less appropriate for sensitivity comparisons.

2. Although a difference in procedure did exist, its impact on the results can be determined quantitatively. Barlow and Kaplan (1977) waited for both the fast and slow phases of dark adaptation (see Wasserman, 1975, for a review of these phases in Limulus) to be "fully" completed between flashes. But Wang-Bennett and Wasserman (1991) used a procedure that allowed the fast phase to be com- 
pleted, and they strictly regulated the adaptive state thereafter (using constant check stimuli) at a level that was slightly above full dark adaptation. Hence the sensitivity of the excised preparation was actually reduced by about a $\log$ unit relative to the maximum possible.

3 . To convert the light measurements in both experiments to a common metric, it was necessary to compute the absorption of the black pigment that shields the receptors. Knowledge of the exact value of this absorption is incomplete; it was taken to have an absorption of $90 \%$ (or 1 log unit) because the pigment is visibly black-since known materials that absorb $90 \%$ of the incident light appear visibly black, the absorption of the shielding pigment therefore cannot be appreciably less than $1 \log$ unit. However, it could be much greater without making the visible appearance of the pigment appreciably blacker.

To be absolutely clear on this point, the sensitivity of the excised data is given in Figure 1 at a virtual lower bound; if pigment absorption were actually greater than $1 \log$ unit, if adaptation were more complete in the excised eye, or if the plateau were used to measure the response, then the intensity-response function of the excised eye would lie to the left of the in situ function by some log units.

It is therefore obvious that the intact eye is not more sensitive than the excised eye when like is compared with like. A similar analysis would demonstrate that the same is true of action potentials recorded from afferent axons. The latter analysis would emerge from a comparison of data such as those shown in the bottom panel of Figure 6 of Wang and Wasserman (1985) with the data shown in Figure 2 of Barlow and Kaplan (1971).

Given the tremendous practical advantages of the excised Limulus lateral eye-particularly its freedom from mechanical vibrations caused by cardiac pulsation, gill cleaning, and exoskeletal movements-there is no reason to conclude that it is a compromised preparation. When like is compared with like, there is no evidence that the sensitivity of the excised eye is less than that of cells recorded in situ. Indeed, recordings from an excised eye may perhaps be even more sensitive than comparable recordings made in situ (because less mechanical disruption of the penetration of the membrane by the microelectrode may occur). The excised eye of Limulus is therefore a unique resource for psychobiological investigation.

\section{REFERENCES}

Barlow, R. B., JR., Chamberlain, S. C., \& KaAs, L. (1984). Circadian thythms in visual function. In S. R. Hilfer \& J. B. Sheffield (Eds.), Molecular and cellular basis of visual acuity (pp. 31-53). New York: Springer-Verlag.

Barlow, R. B., JR., \& Kaplan, E. (1971). Limulus lateral eye: Properties of receptor units in the unexcised eye. Science, 174, 1027-1029.

Barlow, R. B., JR., \& Kaplan, E. (1977). Properties of visual cells in the lateral eye of Limulus in situ: Intracellular recordings. Journal of General Physiology, 69, 203-220.

Barlow, R. B., Jr., Kaplan, E., Renninger, \& Saito, T. (1987). Circadian rhythms in Limulus photoreceptors: I. Intracellular studies. Journal of General Physiology, 89, 353-378.

WANG, L. T., \& Wasserman, G. S. (1980). Effect of adaptation on photoreceptor response duality in Limulus. Society for Neuroscience Abstracts, 6, 194.

Wang, L. T., \& Wasserman, G. S. (1985). Direct intracellular measurement of nonlinear postreceptor transfer functions in dark and light adaptation in Limulus. Brain Research, 328, 41-50.

WANG-BENNETT, L. T., \& WAsserman, G. S. (1991). Visual cells in excised Limulus eyes: Dark adaptation reveals evidence of response duality. Bulletin of the Psychonomic Society, 29, 75-78.

Wasserman, G. S. (1975). Limulus psychophysics: Dark adaptation in the ventral eye. Journal of Experimental Psychology: Human Perception \& Performance, 104, 68-76.

Wasserman, G. S. (1991). Time and duration: A persistent illusion. Perception \& Psychophysics, 50, 603-604.

WASSERMAN, G. S., \& KoNG, K.-L. (1974). Illusory correlation of brightness enhancement and transients in the nervous system. Science, 184, 911-913.

(Manuscript received September 4, 1991.) 